\title{
Validation of rat reference genes for improved quantitative gene expression analysis using low density arrays
}

\author{
Jenny Hong Cai, Shibing Deng, Steven W. Kumpf, Patricia A. Lee, Panayiotis Zagouras, \\ Anne Ryan, and Dan S. Gallagher
}

BioTechniques 42:503-512 (April 2007)

doi 10.2144/000112400

\begin{abstract}
Real-time PCR has become increasingly important in gene expression profiling research, and it is widely agreed that normalized data are required for accurate estimates of messenger RNA (mRNA) expression. With increased gene expression profiling in preclinical research and toxicogenomics, a need for reference genes in the rat has emerged, and the studies in this area have not yet been thoroughly evaluated. The purpose of our study was to evaluate a panel of rat reference genes for variation of gene expression in different tissue types. We selected 48 known target genes based on their putative invariability. The gene expression of all targets was examined in 11 types of rat tissues using TaqMan ${ }^{\circledR}$ low density array (LDA) technology. The variability of each gene was assessed using a two-step statistical model. The analysis of mean expression using multiple reference genes was shown to provide accurate and reliable normalized expression data. The least five variable genes from each specific tissue were recommended for future tissue-specific studies. Finally, a subset of investigated rat reference genes showing the least variation is recommended for further evaluation using the LDA platform. Our work should considerably enhance a researcher's ability to simply and efficiently identify appropriate reference genes for given experiments.
\end{abstract}

\section{INTRODUCTION}

Understanding gene expression patterns may provide insight into complex biological and pathological processes, as well as be predictive of disease outcome or therapeutic treatment $(1,2)$. In recent years, microarray and real-time reverse transcription PCR (RT-PCR) analyses have gained popularity in evaluating messenger RNA (mRNA) expression. Microarray analysis is a genome-wide screening assay based on competitive dual-color hybridization that results in the simultaneous interrogation of thousands of mRNA species. Although microarray analysis is a powerful screening tool for establishing mRNA expression patterns, the extensive replicate sampling can be labor-intensive, sensitivity and dynamic range are small, and analysis of thousands of data points can be technically challenging. The high sensitivity, reproducibility, and large dynamic range of RT-PCR provides highthroughput and accurate differential expression profiling of usually 10-20 select genes $(3,4)$. RT-PCR is extensively applied to functional genomics, molecular medicine, diagnostics, forensics, virology, microbiology, and other biotechnology applications when simultaneous measurement of gene expression in many different samples from small amounts of starting material is required.

Although quantitative RT-PCR is a powerful tool in mRNA expression analysis, there are several variables that need to be controlled, such as RNA quality and quantity and enzyme efficiencies. Therefore, mRNA expression data are often normalized to internal reference genes. Some housekeeping genes are used as reference controls for RT-PCR because they tend to be ubiquitously expressed (5-7). Since the expression of the target gene is normalized to such reference genes, it is essential to choose the appropriate reference gene(s) for accurate and reliable data analysis (8-10).

Commonly used housekeeping genes in real-time RT-PCR assays are $\beta$-actin $(A C T B)$, glycerolaldehydphosphate dehydrogenase (GAPDH), ribosome small subunit (I8S) ribosomal RNA (rRNA), $\beta-2$ microglobulin $\left(B_{2} M\right)$, and hypoxanthine phosphoribosyltransferase $(H P R T)(7,10)$. It is reported that the expression of housekeeping genes can vary considerably under experimental conditions and thus pose problems when interpreting expression data $(3,6,11)$. Suzuki and colleagues discussed the advantages and pitfalls of GAPDH and $\beta$-actin as control genes and emphasized precautions associated with using these as normalizers (12). Vandesompele et al. showed that normalization based on a single housekeeping gene led to erroneous quantification with gene expression changes varying up to 3- 


\section{Research Reports}

fold in $25 \%$ of cases and 6.4-fold in $10 \%$ of cases, while sporadic cases showed errors greater than 20 -fold (13). $18 S$ rRNA shows high sequence conservation among eukaryotes and prokaryotes and is relatively abundant compared with most other mRNA transcripts. This high abundance can make it difficult to accurately subtract the baseline value in real-time RT-PCR data analysis, therefore attenuation of the concentration of $18 \mathrm{~S}$ primers/probe might be needed when quantification of weakly expressed genes is conducted $(6,11,14)$. Without appropriate normalization, expression profiles of target genes will likely be misrepresented (15). With increased gene expression profiling in preclinical research and toxicogenomics, a need for reference genes in rat has emerged; however, extensive studies in this area have not yet been conducted.

In this study, we selected 48 target genes based on putative invariability and examined their expression patterns in 11 rat tissues using the low density array (LDA) platform from Applied Biosystems. This platform allows the simultaneous assay of mRNA gene expression of up to 384 targets on a single card using only a small amount of RNA sample input and a fast setup procedure, therefore large numbers of transcripts can be expeditiously investigated and assessed relatively simply.

\section{MATERIALS AND METHODS}

\section{Tissue Collection, RNA Preparation, and cDNA Synthesis}

Tissues from normal adult rats (three males, three females) were evaluated in this study. Following $\mathrm{CO}_{2}$ asphyxiation and exsanguinations, animals were sacrificed in compliance with The Institutional Animal Care and Use Committee. Tissues were snap-frozen in liquid nitrogen and then stored at $-80^{\circ} \mathrm{C}$. Collected tissues included liver, adrenals, kidney, spleen, jejunum, thymus, lung, heart, brain, gastrocnemius muscle, pancreas, testis, and ovaries for a total of 72 test samples (six animals by eleven common tissues and two sex-related tissues). To insure that tissue samples were collected expedi- tiously, the six animals were processed sequentially and to completion, and the problematic pancreatic tissue was always taken first. Total RNA was extracted from $30 \mathrm{mg}$ each tissue using the RNeasy ${ }^{\circledR}$ Mini kit (Qiagen, Valencia, CA, USA). The tissues were first homogenized using the MagNA Lyser Green Bead tube (Roche Diagnostics, Indianapolis, IN, USA) with $1 \mathrm{~mL}$ icecold lysis buffer on a FastPrep FP120 homogenizer (Thermo Fisher Scientific, Waltham, MA, USA) three times (40 $\mathrm{s}$ at max speed). A QIAshredder ${ }^{\mathrm{TM}}$ (Qiagen) was used to filter the homogenate to prevent clogging on the RNeasy column, and then the manufacturer's recommended RNeasy protocol was followed to completion. All of the RNA samples were treated with DNase as the standard protocol at room temperature for $15 \mathrm{~min}$. RNA was then quantified using the NanoDrop ${ }^{\circledR}$ ND-1000 UVVIS spectrophotometer (Agilent Technologies, Santa Clara, CA, USA). RNA quality was assessed on the Agilent 2100 Bioanalyzer with the RNA 6000 Nano LabChip ${ }^{\circledR}$ kit (Agilent Technologies), and the RNA integrity number (RIN) was calculated based on the entire electrophoretic trace of the RNA sample, including the presence or absence of degradation products (16). RNA is considered to be of high quality if no degradation products are observed in the electrophoretic trace. Subsequently, $1 \mu \mathrm{g}$ high-quality total RNA from samples was reversetranscribed to cDNA using BD Sprint ${ }^{\mathrm{TM}}$ PowerScript $^{\mathrm{TM}}$ Hexamer PrePrimed $6 \times 8$ well (BD Biosciences, San Jose, CA, USA) in $20 \mu \mathrm{L}$ volume at $42^{\circ} \mathrm{C}$ for $90 \mathrm{~min}$ followed by $70^{\circ} \mathrm{C}$ for $10 \mathrm{~min}$ to inactivate the reverse transcriptase, according to the supplier's protocol. One hundred nanograms reversedtranscribed RNA were then loaded into each LDA port and used in real-time PCR assays.

\section{Target Selection}

An internal bioinformatics search [body map and GeneChip ${ }^{\circledR}$ data (Affymetrix, Santa Clara, CA, USA) on human and rat control tissues] identified several genes with low mRNA variability. Additionally, targets were selected from the literature among housekeeping and reference genes commonly used to normalize mRNA expression data. A total of 48 genes were selected and spanned a range of expression levels (high, medium, and low) in a given tissue.

\section{$\operatorname{TaqMan}^{\circledR}$ LDA}

TaqMan LDA microfluidic card technology from Applied Biosystems (Foster City, CA, USA) allows the simultaneous assay of mRNA gene expression of up to 384 targets on a single card. The LDA used in this study was custom designed to consist of 48 TaqMan Gene Expression Assays (Applied Biosystems) per loading port $(48$ genes $\times 8$ samples $=384)$. Each reaction well contained all reagents specific for a given assay. Each target assay consisted of a forward primer, a reverse primer, and a TaqMan MGB probe (6-FAM dye-labeled) and are detailed in Table 1. Although most of the gene assays (36 out of 48) target exon-exon junctions to be mRNAspecific, there are some assays that amplify genomic DNA (12 out of 48). Therefore, we treated all RNA samples with DNase. Several other controls were examined in these assays. No reverse transcriptase negative controls were performed for each RNA sample, to ensure that genomic DNA was removed and not amplified. The positive control was a commercial rat total RNA (BD Rat Universal reference total RNA, $1 \mu \mathrm{g} / \mu \mathrm{L}$; BD Biosciences) and was reverse-transcribed to cDNA along with the test samples. The negative controls consisted of no template (water).

For each tissue sample, $100 \mathrm{ng}$ reverse-transcribed RNA were diluted to $50 \mu \mathrm{L}$ with sterile water, combined with an equal volume of TaqMan Universal PCR Master Mix (2×; Applied Biosystems), mixed by inversion, and spun briefly in an Eppendorf ${ }^{\circledR}$ 5415C microcentrifuge (Brinkmann Instruments, Westbury, NY, USA). After TaqMan LDAs were brought to room temperature, $100 \mu \mathrm{L}$ master mix were loaded into each port connected to 48 reaction wells. LDAs were placed in Sorvall ${ }^{\circledR} /$ Heraeus ${ }^{\circledR}$ Custom Buckets (Applied Biosystems) and centrifuged in a Sorvall Legend ${ }^{\mathrm{TM}}$ centrifuge (Kendro Scientific, Asheville, NC, USA) for 


\section{Research Reports}

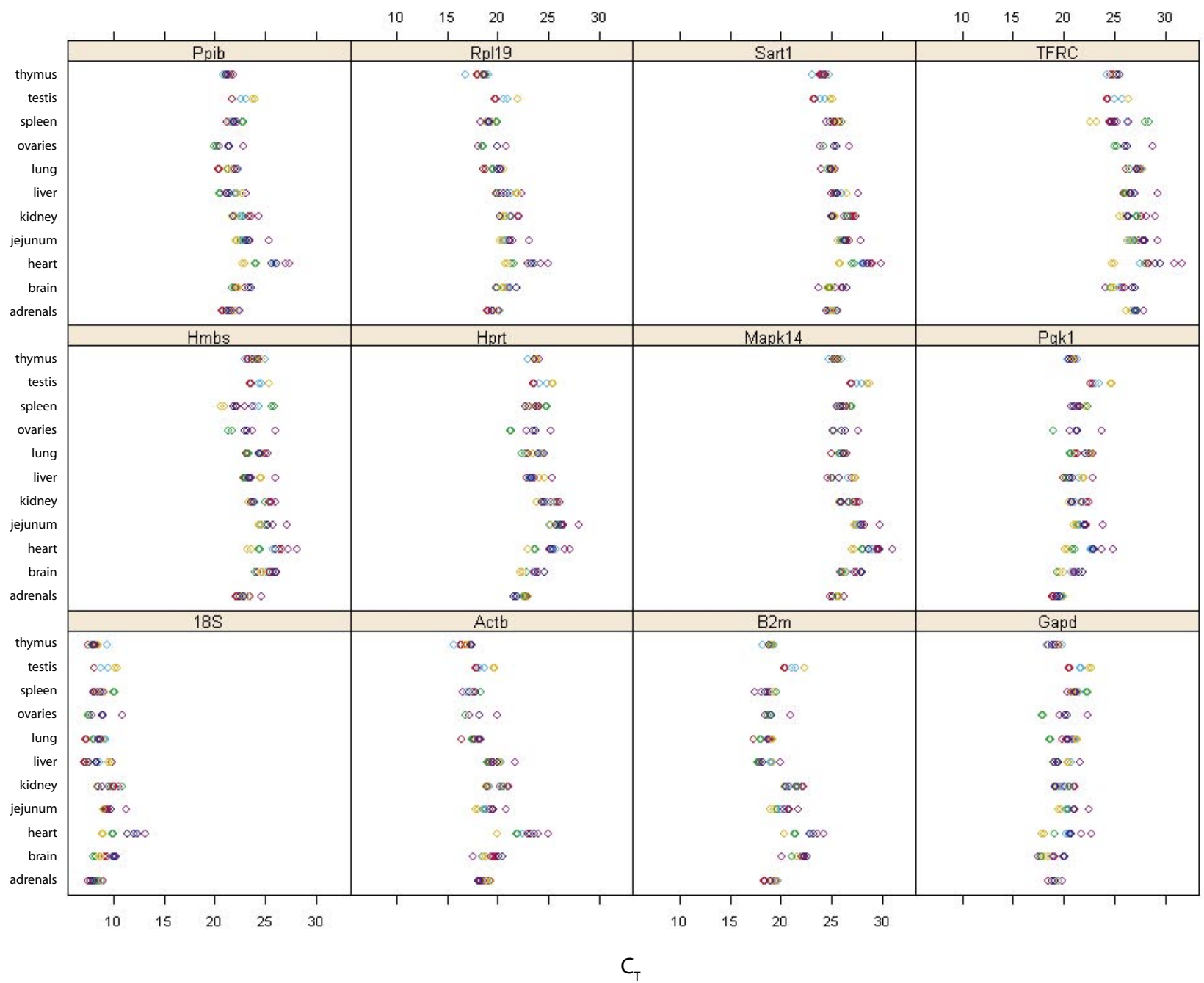

Figure 1. Strip plots representing cycle threshold $\left(C_{T}\right)$ values for tissues and messenger RNA (mRNA) targets for 12 selected genes. The plots representing the remaining 36 genes can be found in Supplementary Figure S1 available online at www.BioTechniques.com. Each circle represents a $\mathrm{C}_{\mathrm{T}}$ value from a sample replicate, so there are 12 circles for each tissue (six animals times two replicates, but six data points for sex tissues), unless data was treated as missing because the sample $\mathrm{C}_{\mathrm{T}}$ values exceeded 40 . Each color represents an animal. Through visual inspection of the strip plots, it is clear that certain mRNA targets show greater tissue variability than do others. Additionally, variability among animals and between replicates is evident.

1 min at $331 \times g$ followed closely by a second 1-min centrifugation at $331 \times$ g. Cards with excess sample in the fill reservoir were spun for an additional 1 min. Immediately following centrifugation, the cards were sealed with a TaqMan LDA Stylus Staker (Applied Biosystems), and the loading ports excised. The final volume in each well after centrifugation was $<1.5 \mu \mathrm{L}$; thus, the final reverse-transcribed RNA concentration was approximately 1.5 ng/reaction. Real-time RT-PCR amplifications were run on an ABI PRISM ${ }^{\circledR}$ 7900HT Sequence Detection System (Applied Biosystems) with a TaqMan LDA cycling block and an automation accessory upgrade. Thermal cycling conditions were $2 \mathrm{~min}$ at $50^{\circ} \mathrm{C}, 10$ min at $95^{\circ} \mathrm{C}$, followed by 40 cycles of denaturation at $95^{\circ} \mathrm{C}$ for $15 \mathrm{~s}$ and annealing and extension at $60^{\circ} \mathrm{C}$ for 1 $\mathrm{min}$. Each test sample was processed in duplicate on individual LDA cards, thus allowing four samples to be processed on each card. There were a total of 20 LDA cards processed in three runs, and controls were included in each of the runs.

\section{Analysis of Real-Time RT-PCR Data}

RT-PCR TaqMan instrumentation monitors gene-specific products with fluorescent dye chemistry. A cycle threshold $\left(\mathrm{C}_{\mathrm{T}}\right)$ for each reaction is the number of cycles at which the reaction crosses a selected threshold. The threshold is defined as a straight line drawn above noise/baseline and positioned within the linear region of the semi-log amplification plot. The fewer cycles required to reach threshold fluorescence intensity, the lower the $\mathrm{C}_{\mathrm{T}}$ value and the greater the initial amount of input target (3). All samples for a given detector were analyzed concurrently using the ABI Relative Quantity Manager software (Applied Biosystems) automated algorithms for background, baseline, and threshold detection (see ABI 7900 User's Guide and Reference 5). Manual confirmation of threshold detection was conducted 


\section{Research Reports}

for quality control purposes. We utilized $\mathrm{C}_{\mathrm{T}}$ number as input for our variability analysis among tissue samples for each target. Since we conducted 40 cycles of PCR, assays that did not yield a $\mathrm{C}_{\mathrm{T}}<40$ cycles were treated as negative results and not included in further analysis. Results for each target on LDAs were quantified concurrently using the same baseline and threshold for a target gene in order to limit interplate errors in the analysis.

Variability of $\mathrm{C}_{\mathrm{T}}$ values was first examined through graphical visualizations (see Figure 2). To quantitatively assess the variability in the $C_{T}$ data, different sources of variability were identified and fitted into a linear random effect model as described below. Based on the design of the experiment, variability was partitioned among animals, tissues, and replicates for a given target. Equation 1 represents the components that contribute to the observed $\mathrm{C}_{\mathrm{T}}$ values

$$
C t_{i j k \mathrm{lg}}=\mu_{g}+C_{k}+T_{i g}+A_{j g(i)}+e_{i j k \mathrm{lg}}
$$

[Eq. 1]

where $C t_{i j k l g}$ is the observed $\mathrm{C}_{\mathrm{T}}$ value of the $i$ th tissue, $j$ th animal, $k$ th card, and $l$ th replicate for the gene $g$. Parameter $\mu_{g}$ is the intercept, the true $\mathrm{C}_{\mathrm{T}}$ value of gene $g$ for a reference group. All parameters are gene-specific except $C_{k}$. $C_{k}$ is a card-specific effect that is added to all genes on the same card and is a systematic effect for which we would like to adjust through normalization. $T_{i g}$ is the effect of tissue $i, A_{j g(i)}$ is the effect of animal $j$ for tissue $i$, and $e_{i j k l \mathrm{lg}}$ is the random error for the $l$ th replication.

The card-specific variability $C_{k}$ explains a small part (due to card) of the overall systematic variability that the reference control genes are intended to remove. The systematic variability due to sample is still included in the genespecific sample terms in Equation 1. Since the systematic sample variability will be the same for all genes, it should have very limited effect for the comparison of the gene-specific sample variability's between genes.

The model in Equation 1 is commonly fitted with a three-way analysis of variance (ANOVA) model where effects are assumed to be fixed parameters, and the variability of each factor is derived from the sum

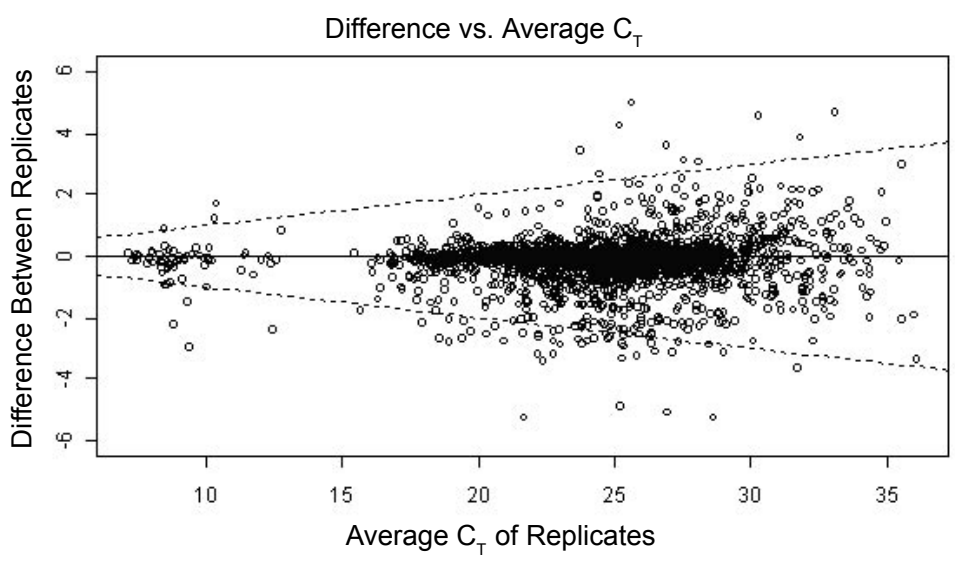

Figure 2. Difference versus mean cycle threshold $\left(\mathbf{C}_{\mathrm{T}}\right)$ of replicates. The dashed lines correspond to a coefficient of variation (CV) of $10 \%$.

of squares associated with each term. However, this fixed effect model does not provide a direct estimate of variability associated with each term; it either depends on the order of the effect entered in the model or does not sum up to the total. So, we fit Equation 1 with a two-step approach, similar to what Littell, Wolfinger, and coworkers (17) used for microarray gene expression data. In the first step, a fixed effect model 1 is fitted to obtain the card-specific effect estimate $\hat{C}_{k}$, and subsequently the card-specific effect is removed from the data to obtain a card-normalized $\mathrm{C}_{\mathrm{T}}$ value $C t^{\prime}{ }_{i j k \lg }$

$$
C t_{i j k \lg }^{\prime}=C t_{i j k \lg }-\hat{C}_{k}
$$

In the second step, we fit a random effect model to the normalized $\mathrm{C}_{\mathrm{T}}$ values for each gene target separately.

$$
C t_{i j k \mathrm{lg}}^{\prime}=\mu_{g}+T_{i g}+A_{j g(i)}+e_{i j k \mathrm{lg}}
$$

[Eq. 3]

Effects $A$ and $T$ are assumed from normal distributions, $T_{i g} \sim$ $N\left(0, \sigma_{T g}^{2}\right)$ and $A_{j g(i)} \sim N\left(0, \sigma_{A g}^{2}\right)$. The error $e$ is assumed to be normally distributed, $e_{i j k \lg } \sim N\left(0, \sigma_{g}^{2}\right)$. The parameters $\sigma_{A g}^{2}$, $\sigma_{T g}^{2}$, and $\sigma_{g}^{2}$ in this model describe the variability associated with animal, tissue, and replicate effect, respectively, and are estimated using the restricted maximum likelihood (REML) method (17). Furthermore, we assume that the terms in Equation 3 are independent of one another. Under these assumptions, the variability of $\mathrm{C}_{\mathrm{T}}$ is a sum of variability of all its components.

$$
\operatorname{Var}\left(C t_{i j k \lg }^{\prime}\right)=\sigma_{A g}^{2}+\sigma_{T g}^{2}+\sigma_{g}^{2}
$$

[Eq. 4]

Compared with a fixed effect model approach, the random effect model has several advantages: $(i)$ it provides a direct estimate of the variability from each source; (ii) it does not depend on how the effects are ordered in the model; and (iii) the total variability is the sum of variability as in Equation 4. Although the assumption of normality may not hold for some effects, in those cases the estimated variance parameters still provide a descriptive measure of the variability associated with the effects. Random effect models are fitted using SAS ${ }^{\circledR}$ PROC MIXED.

\section{RESULTS}

\section{RNA Quality and Yield}

Most RNA samples from rat tissues in this study were of high quality and yield with the exceptions of pancreas and gastrocnemius muscle (data not shown). Most of the tissue samples generated a range of $20-100 \mu \mathrm{g}$ total RNA from $30 \mathrm{mg}$ of each tissue. The 260/280 ratios of the RNA samples were approximately 2, and RIN numbers were near 9 for most samples. RIN normally ranges from 1-10, with an RIN above 7 considered to be indicative of high-quality RNA. All RNA samples from pancreas were extremely degraded (RIN was around 2); this is a common issue in this tissue due to its inherently high RNase activity. RNA quality from gastrocnemius muscle was 


\section{Research Reports}

high, but yield was low ( $<1 \mu \mathrm{g}$ RNA for most samples). This was most likely due to difficulties in tissue lysis from incomplete disruption of the connective components in this tissue and thus made it impossible to fairly evaluate the expression of all 48 gene targets. Therefore, the pancreas and gastrocnemius muscle samples were excluded from comparative expression analysis. In all of the no reverse transcriptase control reactions, no amplification of the 48 gene targets was measured, implying a lack of contribution of genomic DNA to the final expression data.

\section{mRNA Expression Analysis}

Expression analysis was conducted on the remaining 60 samples from 11 tissues and 6 animals (two tissues are sex-specific). The RT-PCR expression data for the 48 genes were acquired and quantified as described. The strip plot (Figure 1) illustrates the dynamic range and variability of $\mathrm{C}_{\mathrm{T}}$ measurements among samples and animals for all mRNA targets. The display of $\mathrm{C}_{\mathrm{T}}$ values indicates that certain genes have greater expression variability among tissues than do others.

To examine the reproducibility of our results, duplicates of each sample were run simultaneously on the same card. Figure 2 displays the difference in $\mathrm{C}_{\mathrm{T}}$ values of replicates relative to their means, most of which $(>95 \%$ of data) fall within a $10 \%$ coefficient of variation $(\mathrm{CV})$. This is an acceptable level of intra-assay variability, therefore LDA assay precision was considered high for the 48 mRNA targets (18). $\mathrm{C}_{\mathrm{T}}$ values of 40 (e.g., some data from Aqp2, Hspa4, S100a3) were treated as missing data and excluded from further analysis.

A two-step modeling approach was used to evaluate sources of variability. Originally we included a gene-specific gender effect in the model, since there are three male and three female rats used in the study. The results indicate a very small gender effect for all mRNAs (data not shown). The variability of $\mathrm{C}_{\mathrm{T}}$ values due to gender is $<1 \%$ of the variability due to tissues for almost all mRNAs when comparing the sum of squares in a fixed effect model. The variability estimate of the gender effect is mostly zero or close to zero in the random effect model. Therefore, the negligible genespecific sex effect was not included in the model for simplicity. Figure 3 represents the variance component estimation based on normal theory for each of the 48 mRNAs with total $C_{T}$ variance partitioned among the three sources and represented in bar graph format. The tissue component accounted for the majority of variability in the data for a given mRNA. In most cases, the animal component accounted for the second most variability followed by the replicate component (tissues $>$ animals $>$ replicates). The fact that the replicate component contributed relatively little to the total variance for a given mRNA further confirmed acceptable assay precision (19). These results are consistent with the strip plots (Figure 1) that visually depict the raw data. Most genes have comparable overall variability, with standard deviations between 1 and 2 (data not shown). The majority of genes display medium levels of expression with $\mathrm{C}_{\mathrm{T}}$ values between 20 and 30 . The $18 S$ mRNA is the most abundant and least variable target. In contrast, S100a3 shows low variability but also low abundance. As would be expected with low expresser targets

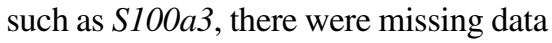
points for some of the tissues.

As an indirect way to validate our approach above, we grouped the 48 genes into three equal-sized groups based on their tissue $C_{T}$ variability. The first group has the 16 genes with smallest tissue variability, and the third group has the largest tissue variability, with the second group in between. If the three groups of genes are used as an endogenous control (pooled for each group) to normalize all data, the $\Delta \mathrm{C}_{\mathrm{T}}$ variability of the 48 genes should be the smallest when the first group is used as the normalizer, while the $\Delta \mathrm{C}_{\mathrm{T}}$ variability should be largest if the data are normalized by the third group. When the three groups of genes are used as normalizers, the median standard deviations of $\Delta \mathrm{C}_{\mathrm{T}}$ of all genes are 1.18, 1.31 , and 2.94, respectively, which in some degree validates our assessment of variability in the housekeeping genes based on their $\mathrm{C}_{\mathrm{T}}$ values.

The level of expression among putative endogenous controls varies widely in different tissues and therefore presents a problem for data analysis. For example, examination of the average $C_{T}$ values among the 48 targets (Figure 3 ) reveals an approximate 16,000-fold difference between the most $(18 S)$ and least abundant (S100a3) transcripts. Furthermore, examination of the strip plots (Figure 1) indicates that some mRNAs are expressed at a relatively high and constant levels in select tissues (e.g., Alb:liver; Gfap:brain; Aqp2:kidney) while variable and low expressing in other tissues. Some of this variability may be due to systematic sample variability; however, the majority is not, because other genes do not show similar changes among these tissues.

\section{DISCUSSION}

There are a number of reviews and research papers evaluating the selection and effect of controls on normalized gene expression data, however, most of them derive from expression analysis in human samples $(4,7,15)$. With increased gene expression profiling in preclinical research and toxicogenomics, there is an urgent need for rigorous validation of reference genes in experimental animal model systems. A recent paper has discussed the validation of canine reference genes for gene expression in that species (20). The study of rat reference genes for gene expression has not yet been thoroughly evaluated. Routinely, high-quality quantitative gene expression data are often normalized relative to a reference control gene. Therefore, it is critical to choose an appropriate reference control gene(s) for normalization so as not to misrepresent the expression profile of a target gene (11). Since no single reference gene is optimal for all studies, the selection of an appropriate control gene for a given study is key, especially among complex multiple tissue and treatment regimens (9). In our study, we evaluated the expression levels for 48 genes across a panel of rat tissues to select potential reference genes for normalization of mRNA expression data.

The first step in any mRNA expression analysis study is to confirm the quality and yield of RNA from the 


\section{Research Reports}

Table 1. 48 mRNA Targets Included on the Low Density Array

\begin{tabular}{|c|c|c|c|c|c|}
\hline $\begin{array}{l}\text { Gene } \\
\text { No. }\end{array}$ & $\begin{array}{l}\text { Gene } \\
\text { Symbol }\end{array}$ & Gene Name & Accession No. & $\begin{array}{l}\text { Assay } \\
\text { Location } \\
\text { (nt) }\end{array}$ & $\begin{array}{l}\text { ABI Gene Expression } \\
\text { Assay No. }\end{array}$ \\
\hline 1 & FLJ20445 & Hypothetical protein & XM_215286 & 1100 & Rn01454146_m1 \\
\hline 3 & FLJ10587 & Hypothetical protein & BF411321 & 2400 & Rn01456650_m1 \\
\hline 4 & COP9 & $\begin{array}{l}\text { COP9 constitutive photomorphogenic homolog sub- } \\
\text { unit } 6 \text { (Arabidopsis) }\end{array}$ & $\begin{array}{l}\text { XM_222002; } \\
\text { Al409481 }\end{array}$ & 589 & Rn01528050_g1 \\
\hline 6 & Hspa4 & Likely ortholog of mouse heat shock protein, $70 \mathrm{kDa} 4$ & BE101732 & 439 & Rn01477779_m1 \\
\hline 7 & FLJ10498 & Hypothetical protein & BF394953 & 388 & Rn01414061_m1 \\
\hline 8 & Epo & Brain Zn-finger protein (LOC362154) & BF550329 & 463 & Rn01515220_g1 \\
\hline 9 & AP82 & Acidic $82 \mathrm{kDa}$ protein mRNA & BF410997 & 1551 & Rn01521658_m1 \\
\hline 10 & Ring1 & Ring finger protein 1 & $\mathrm{BI} 300772$ & 1041 & Rn01429212_g1 \\
\hline 13 & Stau & Staufen & NM_053436 & 169 & Rn00584855_m1 \\
\hline 14 & GAPDH & Glyceraldehyde-3-phosphate dehydrogenase & $\mathrm{X} 02231$ & $327-377$ & Rn99999916_s1 \\
\hline 15 & RPL10a & Ribosomal protein L10A & NM_031065 & 334 & Rn00821239_g1 \\
\hline 16 & Ppib & Cyclophilin B & NM_022536 & 251 & Rn00574762_m1 \\
\hline 17 & L32 & Ribosomal protein L32(Rpl32) & est594385; X06483 & 421 & Rn00820748_g1 \\
\hline 18 & PPIA & Peptidylprolyl isomerase A (Cyclophilin A) & M19533 & 228 & Rn00690933_m1 \\
\hline 19 & ActB & Actin, $\beta$ & NM_031144 & 888 & Rn00667869_m1 \\
\hline 20 & RPL19 & Ribosomal protein L19 & $\begin{array}{l}\text { AA800054; } \\
\text { NM_031103 }\end{array}$ & 266 & Rn00821265_g1 \\
\hline 21 & Nedd4a & $\begin{array}{l}\text { Neural precursor cell expressed, developmentally } \\
\text { down-regulated gene } 4 \mathrm{~A}\end{array}$ & U50842 & 2564 & Rn01530544_m1 \\
\hline 29 & $B 2 m$ & $\beta-2$ microglobulin & NM_012512 & 71 & Rn00560865_m1 \\
\hline 30 & Jak2 & Janus kinase 2 & NM_031514 & 133 & Rn00580452_m1 \\
\hline 31 & Gusb & Glucuronidase, $\beta$ & NM_017015 & 1390 & Rn00566655_m1 \\
\hline 32 & Map3k1 & Mitogen activated protein kinase kinase kinase 1 & NM_053887 & 1316 & Rn00588007_m1 \\
\hline 33 & Pdeзa & Phosphodiesterase $3 \mathrm{~A}$ & NM_017337 & 1004 & Rn00569192_m1 \\
\hline 34 & Mapk6 & Mitogen-activated protein kinase 6 & NM-031622 & 1089 & Rn00581152_m1 \\
\hline 35 & S100a3 & S100 calcium binding protein A3 & NM_053681 & 39 & Rn00586633_m1 \\
\hline 36 & Rpn1 & Ribophorin I & NM_013067 & 328 & Rn00565052_m1 \\
\hline 37 & Tabu1 & Tubulin & NM_022298 & 71 & Rn01532518_g1 \\
\hline 38 & TFRC & Transferrin receptor & rCT52708 & 569 & Rn01474701_m1 \\
\hline 39 & HPRT1 & Hypoxanthine guanine phosphoribosyl transferase & M63983 & 486 & Rn01527838_g1 \\
\hline 40 & Ubd & Ubiquitin D & NM_053229 & 79 & Rn00583977_m1 \\
\hline 41 & $a l b$ & $\alpha$ Albumin & NM_134326 & 716 & Rn00592480_m1 \\
\hline 42 & GFAP & Glial fibrillary acidic protein & NM_017009 & 1189 & Rn00566603_m1 \\
\hline 43 & Tnni1 & Troponin I, slow isoform & NM_017184 & 235 & Rn00567843_m1 \\
\hline 44 & $A Q P 2$ & Aquaporin 2 & NM_012909 & 486 & Rn00563755_m1 \\
\hline 45 & PGK1 & Phosphoglycerate kinase 1 & NM_053291 & 1019 & Rn00821429_g1 \\
\hline 46 & Rp/13a & Ribosomal protein L13A & NM_173340 & 272 & Rn00821946_g1 \\
\hline 47 & TBP & $\begin{array}{l}\text { TAF9-like RNA polymerase II, TATA box binding pro- } \\
\text { tein (TBP)-associated factor, } 31 \mathrm{kDa} \text { (Taf9I ) }\end{array}$ & NM_133615 & 478 & Rn00592425_m1 \\
\hline 48 & $18 S$ & Eukaryotic $18 \mathrm{~S}$ rRNA & X03205 & & Hs99999901_s1 \\
\hline
\end{tabular}




\section{Research Reports}

Variance Estimation

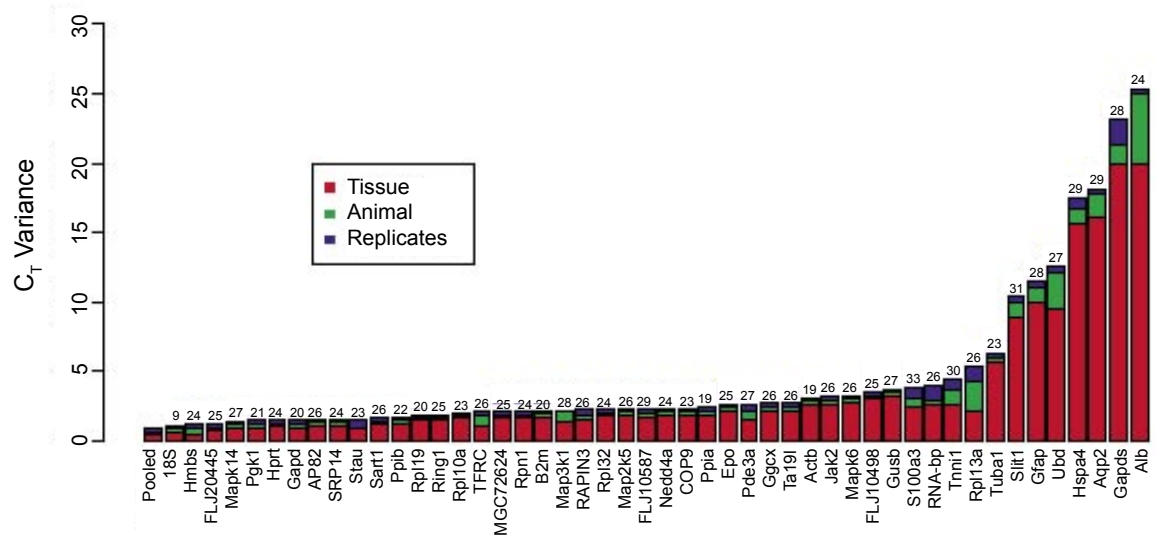

Figure 3. Variance component estimation based on normal theory for each messenger RNA (mRNA). Total cycle threshold $\left(\mathrm{C}_{\mathrm{T}}\right)$ variance is partitioned among three sources. The bar graphs are arranged from left to right to show lowest to highest $\mathrm{C}_{\mathrm{T}}$ variance. Through visual inspection of the data, it is evident that in most cases the majority of $\mathrm{C}_{\mathrm{T}}$ variance is attributable to differences among tissues. The number on top of each bar represents the mean $\mathrm{C}_{\mathrm{T}}$ for all analyzable samples for a given target. The first bar to the left was derived from pooling data among the four mRNA targets showing the lowest total $\mathrm{C}_{\mathrm{T}}$ variance.

processed tissues, because RNA is less stable than DNA in normal working conditions. RNA degradation and transcriptional induction can occur immediately after harvesting of the biological sample (21). Due to poor quality and low yield of RNA, pancreas and gastrocnemius muscle samples were excluded from comparative expression analysis. In the future, alternative extraction methods may be evaluated to improve RNA quality and yield from

Other considerations for accurate real-time PCR also include evaluations of assay efficiency, assay variation, and potential assay inhibition (22). A recent report on the evaluation of LDA technology indicated that gene muscle and pancreas tissues.

expression measurements conducted with LDAs are highly reproducible and precise, both within and across arrays. Comparisons between LDAs reveal low variability, with correlation coefficients close to 1.0 (19). Although our study was not designed to look at the assay inhibition, it is interesting to note the breakdown of the variance analysis by tissue, animal, and replicate-specific components reports indirectly on any influence by an assay inhibitor. Figure 3 clearly shows that the tissue variance outweighs any assay-specific effects.

In normal rat tissues, nearly twothirds of the 48 mRNA targets showed relatively low expression variability among replicates, tissues, and animals; and thus, are considered good candidates

Table 2. Top Five Control Genes Recommended for Each Tissue

\begin{tabular}{|llllll|}
\hline Organ & Selection 1 & Selection 2 & Selection 3 & Selection 4 & Selection 5 \\
\hline Liver & B2M & Pgk1 & Rpn1 & Ggcx & FLJ20445 \\
Kidney & Pgk1 & Ring1 & ActB & Gapd & Mapk14 \\
Brain & Mapk6 & Gapd & Rp10a & TFRC & Map2k5 \\
Heart & Rpl10a & COP9 & Gapd & Ppib & Hmbs \\
Lung & Rpl10a & Mapk14 & FLJ20445 & Taf91 & Map2k5 \\
Spleen & Ppib & Tuba1 & COP9 & Taf91 & Rpl19 \\
Jejunum & FLJ20445 & Rpl10a & MGC72624 & Tuba1 & FLJ10498 \\
Adrenals & Rpl19 & Mapk14 & Nedd4a & FLJ10586 & Ggcx \\
Thymus & Rpl10a & Hprt & Pgk1 & Gusb & COP9 \\
Testis & B2M & FLJ10498 & RABIN3 & Rpl10a & SRP14 \\
Ovaries & Actb & Ppib & COP9 & FLJ20445 & Epo \\
\hline
\end{tabular}

to be selected as reference controls. However, it is impossible to predict how different experimental conditions may affect the expression of putative normalization genes. When measuring gene expression under different experimental conditions (e.g., drug treatments, diseases, etc.), this subset of mRNAs should be further evaluated so as to select the best reference gene(s) for specific experimental conditions. When examining gene expression patterns among multiple tissues/treatment groups, it is imperative that the same normalization strategy be applied to all tissues types, in order to reduce the number of experimental variables and allow direct comparison between samples. In other words, the same set of control genes should be used for normalization among tissues and groups, if possible (13).

We analyzed tissue-specific gene expression profiles and listed the five control genes for each tissue showing the least $\Delta \mathrm{C}_{\mathrm{T}}$ variability within the tissue (Table 2). The rank is based on the standard deviation of $\Delta \mathrm{C}_{\mathrm{T}}$ (data not shown) calculated by normalizing to a pooled average of the 36 genes (see Figure 3) showing the least variable expression in $\mathrm{C}_{\mathrm{T}}$. For example, $B_{2} M$ is a good potential reference gene for liver, but not a good one for heart. This suggests that different reference gene(s) or biomarkers may be used when a given tissue is studied. The reference gene should also be of similar abundance to the gene of interest. For $18 \mathrm{~S}$, which is shown as the most stable gene in the overall variability assessment in Figure 1, but is not in any of the top organ-specific gene lists in Table 2, the abundance is so high that it in fact explains its overall stability. This can also be seen from Figure 1. Assay attenuation of $18 S$ is not possible due to the nature of LDA technology, as all the gene assays are preloaded on the plate and there is no chance to further optimize the abundance of the gene assay to the target gene level. Therefore, $18 S$ is decidedly not the best reference gene within a tissue or in its potential ability to respond to experimental conditions, due to its high abundance.

Normalization based on a single reference gene without validation can lead to erroneous results (11). 


\section{Research Reports}

Different methods for identifying the most suitable combination of control genes have been proposed. Recent studies using the geometric mean and pair-wise comparison from multiple housekeeping genes suggest that to accurately measure gene expression, normalization to multiple housekeeping genes is essential when many target genes are assayed (13). A recent report by de Kok and colleagues details the calculation and application of the mean expression from multiple housekeeping genes (7). Evaluating the approach of using multiple reference controls, our analysis has shown that by pooling the four least variable genes $(18 \mathrm{~S}, \mathrm{Hmbs}$, FLJ20445, Mapk14), variation within the pooled data was less than any one of the four genes (Figure 3). However, underpinning the increased confidence achieved with pooling of a small number of reference genes for normalization is the critical fact that each and every reference control gene should be experimentally validated in the test system under study. Our approach represents a first step in that direction.

Under conditions where samples are limited, such as in an early developing embryo or a minute biopsy sample, it might not be prudent to prescreen experimental material to select a reference gene to use in normalizing expression data. In such a case, preselection of a battery of reference genes known to vary minimally among tissues would reduce the likelihood of the expression levels of target mRNAs being skewed by a single gene, the expression of which varies under the experimental conditions in question. By normalizing across several select reference genes and estimating the inherent component variance, the potential negative impact on the accuracy of relative gene expression data would be minimized. This approach would allow more rapid gene expression data collection in the pharmaceutical setting and supply a downstream data analysis tool to support the selection of the best reference genes for the study in question.

The number of genes used to normalize mRNA expression data are a trade-off between practical considerations and assay validity within an experimental design. For example, it might not be reasonable to use numerous reference controls when only a few mRNAs are to be studied in normal tissues, especially when sample template availability is limiting. If the goal is to select a single normalization gene, the control and mRNA(s) of interest should be expressed at similar levels, and the control should not vary under experimental conditions $(6,11,23)$. However, if the expression of many genes is to be evaluated among numerous tissue and/ or treatment groups, it may then become necessary to assay multiple reference controls for normalization of the data.

Although the primary purpose of this study was to assess the variation of reference control genes in various tissues, the same rules can also be applied to other experimental conditions, such as in vitro assays. We have several in-house programs utilizing our rat reference LDA cards that have successfully uncovered the reference genes that remained stable under each study's complicated conditions. The commonly used housekeeping genes, like $\beta$-actin and $G A P D H$, were not appropriate selections for the experiments in question. We have successfully applied one to six internal controls for gene expression analysis (data not shown) for a number of programs, each based on experimental design. From our experience, we feel strongly that the use of a battery of reference genes within the LDA format not only expedites the selection of custom endogenous controls but also improves the quality of the gene expression data. Therefore, we recommend that the use of the reference control LDA card and the analysis of component variance, within an appropriately normalized real-time experiment, is the first step to confident quantitative RT-PCR gene expression data.

\section{ACKNOWLEDGMENTS}

J.H.C., D.S.G., and A.R. participated in hypothesis design. J.H.C. designed the experiment, and J.H.C., S.W.K., and P.A.L. carried out the experiments. P.Z. provided the bioinformatics search on the invariable genes. S.D. supported all statistical analysis. J.H.C., S.D., and D.S.G. drafted the manuscript. A.R. was a critical reviewer. All authors read and approved the final manuscript. We thank James Yan for contributing the ideas on study design and Gary Seitis for providing necropsy assistance and collecting study tissue materials.

\section{COMPETING INTERESTS STATEMENT}

The authors declare no competing interests.

\section{REFERENCES}

1.Ge, X., S. Yamamoto, S. Tsutsumi, Y. Midorikawa, S. Ihara, S.M. Wang, and H. Aburatani. 2005. Interpreting expression profiles of cancers by genome-wide survey of breadth of expression in normal tissues. Genomics 86:127-141.

2. van't Veer, L.J., H. Dai, M.J. van de Vijver, Y.D. He, A.A.M. Hart, M. Mao, H.L. Peterse, K. van der Kooy, et al. 2002. Gene expression profiling predicts clinical outcome of breast cancer. Nature 415:530-536.

3. Akilesh, S., D.J. Shaffer, and D. Roopenian. 2003. Customized molecular phenotyping by quantitative gene expression and pattern recognition analysis. Genome Res. 13:17191727.

4.Bustin, S. 2002. Quantification of mRNA using real-time reverse transcription PCR (RT-PCR): trends and problems. J. Mol. Endocrinol. 29:23-39.

5.Abruzzo, L., K. Lee, A. Fuller, A. Silverman, M. Keating, L. Medeiros, and K. Coombes. 2005. Validation of oligonucleotide microarray data using microfluidic low-density arrays: a new statistical method to normalize real-time RT-PCR data. BioTechniques 38:785-792.

6. Brunner, A.M., I.A. Yakovlev, and S.H. Strauss. 2004. Validating internal controls for quantitative plant gene expression studies. BMC Plant Biol. 4:14.

7.de Kok, J.B., R.W. Roelofs, B.A. Giesendorf, J.L. Pennings, E.T. Waas, T. Feuth, D.W. Swinkels, and P.N. Span. 2005. Normalization of gene expression measurements in tumor tissues: comparison of 13 endogenous control genes. Lab. Invest 85:154-159.

8. Kim, B.-R., H.-Y. Nam, S.-U. Kim, S.-I. Kim, and Y.-J. Chang. 2003. Normalization of reverse transcription quantitative-PCR with housekeeping genes in rice. Biotechnol. Lett. 25:1869-1872.

9.Schmittgen, T.D. and B.A. Zakrajsek. 2000. Effect of experimental treatment on housekeeping gene expression: validation by real-time, quantitative RT-PCR. J. Biochem. Biophys. Methods 46:69-81.

10. Weisser, M., T. Haferlach, C. Schoch, W. Hiddemann, and S. Schnittger. 2004. The use of housekeeping genes for real-time PCR-based quantification of fusion gene transcripts in acute myeloid leukemia Leukemia 18:1551-1553.

11. Tricarico, C., P. Pinzani, S. Bianchi, M. Paglierani, V. Distante, M. Pazzagli, S.A. Bustin, and C. Orlando. 2002. Quantitative real-time reverse transcription polymerase chain reaction: normalization to rRNA or single housekeeping genes is inappropriate for human tissue biopsies. Anal. Biochem. 309:293-300.

12. Suzuki, T., P.J. Higgins, and D.R. Crawford. 2000. Control selection for RNA quantitation. BioTechniques 29:332-337. 
13. Vandesompele, J., K. De Preter, F. Pattyn, B. Poppe, N. Van Roy, A. De Paepe, and F. Speleman. 2002. Accurate normalization of real-time quantitative RT-PCR data by geometric averaging of multiple internal control genes. Genome Biol. 3:research0034.0031research0034.0011.

14. Smith, R.D., B. Brown, P. Ikonomi, and A.N. Schechter. 2003. Exogenous reference RNA for normalization of real-time quantitative PCR. BioTechniques 34:88-91.

15. Thellin, O., W. Zorzi, B. Lakaye, B. De Borman, B. Coumans, G. Hennen, T. Grisar, A. Igout, et al. 1999. Housekeeping genes as internal standards: use and limits. J. Biotechnol. 75:291-295.

16. Imbeaud, S., E. Graudens, V. Boulanger, $X$. Barlet, P. Zaborski, E. Eveno, O. Mueller A. Schroeder, et al. 2005. Towards standardization of RNA quality assessment using user-independent classifiers of microcapillary electrophoresis traces. Nucleic Acids Res. 33: e56.

17. Littell, R., G.A. Milliken, W.W. Stroup, and R.D. Wolfinger. 1996. SAS system for mixed models, p. 491-504. SAS Publishing, Cary, NC.

18. Food and Drug Administration. 2001 Guidance for Industry, Bioanalytical Method Validation. May, p. 15. U.S. Department of Health and Human Services, Rockville, MD.

19. Goulter, A., D. Harmer, and K. Clark. 2006 Evaluation of low density array technology for quantitative parallel measurement of multiple genes in human tissue. BMC Genomics 7:34.

20. Brinkhof, B., B. Spee, J. Rothuizen, and L.C. Penning. 2006. Development and evaluation of canine reference genes for accurate quantification of gene expression. Anal. Biochem. 356:36-43.

21. Bustin, S.A. and T. Nolan. 2004. Pitfalls of quantitative real-time reverse-transcription polymerase chain reaction. J. Biomol. Tech. 15:155-166.

22. Dheda, K., J.F. Huggett, S.A. Bustin, M.A. Johnson, G. Rook, and A. Zumla. 2004. Validation of housekeeping genes for normalizing RNA expression in real-time PCR BioTechniques 37:112-119.

23. Zhong, H. and J.W. Simons. 1999. Direct comparison of GAPDH, [beta]-actin, cyclophilin, and 28S rRNA as internal standards for quantifying RNA levels under hypoxia. Biochem. Biophys. Res. Commun. 259:523526.

Received 7 September 2006; accepted 15 December 2006

Address correspondence to Jenny Cai, MS 8274-1418, Safety Science, PGRD, Pfizer Inc., Eastern Point Road, Groton, CT 06340, USA.e-mail: jenny.h.cai@pfizer.com

To purchase reprints of this article, contact: Reprints@BioTechniques.com 